\title{
EDITORIAL
}

\section{La feminización en la Medicina}

\author{
Feminization in Medicine
}

\author{
Patrizio Petrone, MD, MPH, MHSA, FACS
}

Director of Surgical Research, Program Director, International Research Fellowship, Department of Surgery - NYU Winthrop Hospital.

Históricamente, las mujeres han desempeñado una función de suma importancia en la prestación de asistencia sanitaria. Así ocurrió en la mayoría de los países con anterioridad a la aparición de los sistemas modernos de atención de salud, siendo las mujeres las principales dispensadoras de cuidados en el seno de las familias y de sus comunidades, y actuando en muchos países en desarrollo como parteras tradicionales. Fuera del ámbito familiar, las mujeres ocupan el primer lugar en las filas del personal voluntario que trabaja en los hospitales y otras organizaciones comunitarias. También lo son en las escuelas elementales entre cuyas tareas se encuentra la enseñanza de conductas favorables para el mantenimiento de la salud y la prevención de enfermedades.

Son muchas las razones que explicarían el por qué de esta función de las mujeres. Una de ellas se debe a las formas de diferenciación de funciones por razón de sexo desde su nacimiento, mediante aprendizaje y condicionamiento social. Dicha diferenciación se observa en una división sexual del trabajo en el seno de la familia y en el mercado laboral, actitud arraigada en las tradiciones y costumbres sociales donde la idea que la división del trabajo entre los miem- bros masculinos y femeninos de la familia es más complementaria que competitiva, y donde los padres sirven como modelos y roles de función. El otro aspecto de esta diferenciación está marcado en las funciones asignadas por el mercado laboral.

A lo largo de la historia, las diferentes profesiones y actividades sanitarias han ido construyendo identidades profesionales sexuadas, las que han ido variando según los momentos históricos. Desde la creación de las universidades en el siglo XIII hasta finales del siglo XIX, cuando las primeras mujeres empezaron a estudiar y a practicar la medicina, los miembros de la profesión eran exclusivamente hombres. Pero la incorporación de actividades codificadas como masculinas, ha sido un proceso activo y cambiante en virtud del cual las habilidades consideradas femeninas se convirtieron en masculinas, como sucedió con la atención del parto en los siglos XVIII y XIX.

En la segunda mitad del siglo XIX, comienza a considerarse que la educación puede preparar a la mujer para cumplir mejor la tarea de formar nuevos ciudadanos y constituir un apoyo adecuado para maridos modernos. Parte de la respuesta a este fenómeno reside en la cultura local de

Palabras clave: feminización; educación médica; docentes médicos; rol del médico; historia.

Key words: feminization; education, medical; physician's role; history.

Fecha recibido: 5/04/2018 Fecha aceptación: 6/04/2018

Correspondencia: Patrizio Petrone, MD, FACS. 222 Station Plaza North, Suite 603, Mineola, Long Island, New York, 11501, USA Correo electrónico: patrizio.petrone@gmail.com

Citar como: Petrone P. La feminización en la Medicina. Rev Colomb Cir. 2018;33:132-34. https://doi.org/10.30944/20117582.54 
aquella época, promulgada por la Iglesia Católica y las escuelas. La prensa femenina apoyaba el consenso local de la cultura del momento acerca que el entorno natural de las mujeres se hallaba limitado al hogar y a la familia. No fue sino hasta la edición de la revista La vie heureuse en 1905, en que comenzaron a publicarse artículos sobre mujeres practicando actividades deportivas, presentando nuevas posibilidades por fuera de la vida doméstica.

A pesar de que aún en la actualidad hay más varones que mujeres en el mundo del trabajo remunerado, la tasa de empleo de las mujeres ha aumentado y la de los varones ha disminuido, denominándose a este proceso como 'feminización del empleo'. El aumento de la participación femenina responde a un proceso de larga duración vinculado fundamentalmente al acceso de las mujeres a mayores niveles educativos y a transformaciones culturales que influyeron en el cambio de las pautas de fecundidad.

El papel de la mujer ha sido relegado por años a tareas domésticas o producción artesanal, otorgándole poca capacidad de investigación, producción y creación en campos como las ciencias. A pesar de la revolución sexual en los años sesenta y la participación de la mujer en la vida productiva, quedan aún algunos peldaños de difícil acceso que obstruyen su carrera hacia el reconocimiento.

A pesar de los cambios en la composición de la fuerza de trabajo sectorial, la persistencia de situaciones de segregaciones vertical (por categorías) y horizontal (por tareas), produce las relaciones típicas de los sectores 'femeninos'. En el caso de segregación vertical, definida como la diferencia entre los potenciales aspirantes a puestos de conducción y quienes efectivamente ocupan dichos cargos, en general, las mujeres no acceden a puestos de conducción, como jefaturas de servicio o direcciones de hospitales. En el mismo sentido, la segregación horizontal se da cuando la demanda de trabajadores mujeres se presenta en cargos y funciones asociadas con tareas reproductivas y que requieran cuidados maternales.
Es relevante considerar que muchas decisiones sobre organizaciones de salud y políticas sanitarias se configuran sin tener en cuenta la naturaleza de los procesos de trabajo. Los esfuerzos por asociar calidad con recursos humanos, promueven una articulación más estrecha entre formación, práctica profesional y capacidad resolutiva de los servicios. Los recursos humanos en salud deben resolver tensiones y conflictos manifiestos, como los intraprofesionales e interprofesionales, las relaciones entre capital y trabajo y entre tabajador y empleador, además de otros más solapados, como las inequidades sociales, de etnia y de sexo, que atraviesan el mundo del trabajo.

$\mathrm{Al}$ focalizarnos en el sector salud, resulta ser hoy el campo de práctica para más de 20 profesiones universitarias, con cerca de Ioo especialidades, alrededor de 40 carreras técnicas y auxiliares, constituyéndose como un espacio de empleo para otros oficios hasta ocupar, en conjunto, casi el $5 \%$ de la población económicamente activa en varios países de la región. Desde la década de los 6o, las universidades latinoamericanas no han parado de generar una mayor dotación de profesionales de la salud. Varios países se encuentran en una situación autoperceptiva que se podría denominar de sobredotación o exceso de profesionales. Sin embargo, otros países tienen hoy un déficit de profesionales médicos, ya sea por su nivel de desarrollo relativo o por falta de planificación.

El estudio de los recursos humanos en salud puede ser enfocado desde diferentes perspectivas, comenzando por la propia demografía profesional que, a partir de la lógica de mercado de trabajo, estudia su oferta y su demanda. De allí se desprenden cifras globales y estimaciones: 7,5 millones de trabajadores de salud en la región de las Américas, cerca de Ioo millones en el mundo.

En I96I, se presentó una iniciativa regional denominada Alianza para el Progreso, cuya agenda promovía un componente social de desarrollo, con educación y salud como prioridades. En el campo educativo, los países de Latinoamérica expandieron su matrícula universitaria en el pe- 
ríodo $1960-1980$, llegando a multiplicar por $20 \mathrm{su}$ población estudiantil, debiéndose gran parte de ese aumento al ingreso de estudiantes mujeres por un doble mecanismo: por un lado, la 'feminización' progresiva de las carreras tradicionales en salud (Medicina, Odontología, Bioquímica y Farmacia), y, por el otro, la incorporación en el ámbito universitario de carreras clásicamente femeninas que hasta ese momento se dictaban en instituciones terciarias (Enfermería, Trabajo Social, Nutrición y Obstetricia).

Durante 1972, en el marco del lanzamiento del Plan Decenal de las Américas, se planteó como meta lograr una relación de un médico por cada I.ooo habitantes. Si bien había sido lanzada seis años antes de Alma Ata, para muchos países constituía una verdadera utopía. Al combinarse dicha meta con el énfasis en ampliar la oferta universitaria, sobre todo de las universidades públicas en Latinoamérica, se impulsó la formación profesional en salud, lo que fue aprovechado particularmente por las estudiantes mujeres, generando un impacto en la composición de la fuerza laboral del sector.

No está del todo claro el efecto de la feminización sobre la fuerza laboral, ya que estudios publicados en los Estados Unidos y Europa demostraron que, si bien las mujeres tienden a trabajar menos horas y a tomar más tiempo de licencia durante el embarazo y el puerperio, también es cierto que, luego del período de crianza de sus hijos, permanecen más tiempo en su carrera profesional y se retiran más tarde que muchos varones. Aunque en la actualidad el ejercicio de la profesión médica plantea a priori una situación de total igualdad en el plano laboral para varones y mujeres, factores sociales, culturales e incluso biológicos, pueden hacer que una situación en principio teóricamente igualitaria desemboque en situaciones que no corresponden con una igualdad real.
Es importante destacar la escasa relación existente entre el conocimiento y la formulación de políticas públicas en varios países de Latinoamérica y, por ende, la importancia técnica y de argumentos de calidad académica que cuente con información desagregada, para aumentar la capacidad de convocatoria a los distintos actores gubernamentales y de la sociedad civil involucrados en el sector salud, para proponer, de forma participativa, informada y consensual, las estrategias y programas que el sector requiere para contribuir al empleo de manera más equitativa, justa y eficiente.

En conclusión, la demografía de las profesiones de la salud está cambiando en Latinoamérica y en el mundo, con una feminización creciente de la profesión médica. Está claro que la feminización ha traído aparejada una serie de ventajas tanto para los pacientes como para los trabajadores de la salud. No obstante, la feminización también viene acompañada de nuevos desafíos que deberán ser abordados oportunamente.

\section{Lecturas recomendadas}

I. Abramzón M. Argentina: recursos humanos en salud en 2004. Buenos Aires: Organización Panamericana de la Salud-Organización Mundial de la Salud; 2005. II2p.

2. Ortiz-Gómez T, Birriel-Salcedo J, Ortega del Olmo R. Género, profesiones sanitarias y salud pública. Gac Sanit. 2004;I8(Supl.I):I89-94.

3. Petrone P. Avances y retrocesos de la feminización en los equipos de salud. Buenos Aires: Editorial Dunken; 20I4. IO4 p.

4. Rovere M. Recursos humanos en salud. Bases para una agenda impostergable. Presentado en la reunión del Plan Fenix II, Proyecto Estratégico de la Facultad de Ciencias Económicas (UBA), 2005.

5. Rovere M. Planificación estratégica de recursos humanos en salud. Washington, D.C.: Organización Panamericana de Salud; 2006. 232 p.

6. Simoens S, Hurst J. The supply of physicians services in OECD countries. OECD Health Working Papers, $\mathrm{N}^{\circ}$ 2I. París: Organisation for Economic Co-operation and Development (OECD); 2006. 62 p. 